\title{
More Tools in the Toolbox: The Naval Oceanographic Office's Remote Environmental Monitoring UnitS (REMUS) 6000 AUV
}

\author{
Kenneth M. Sharp and Randy H. White \\ Naval Oceanographic Office \\ Ocean Projects Department \\ Stennis Space Center, MS 39522-5001 USA
}

\begin{abstract}
Autonomous underwater vehicles (AUVs) are useful and necessary tools for modern oceanographic data collection. The Naval Oceanographic Office (NAVOCEANO) Ocean Projects Department has been successfully applying AUV technology since 1997. NAVOCEANO's entry into the large AUV realm was initiated with the transfer of a vehicle developed and tested at Draper Labs in 1997, named Lazarus. NAVOCEANO also teamed with Penn State Applied Research Laboratory to design and build the SEAHORSEClass AUV, with the first of three vehicles delivered in 2001. These vehicles are powered by D-cell alkaline batteries and were mainly used to develop AUV Concept of Operations and logistic requirements. These vehicles are designed to operate under a preprogrammed set of rules and instructions with the goal of carrying out assigned missions without direct operator interaction or supervision. This concept would provide a "force multiplier" to other NAVOCEANO survey assets. However, in order for AUVs to become operationally effective, several technology gaps needed to be overcome. These gaps included sensors, communications, navigation, power, and launch and retrieval systems. As technologies advanced, the REMUS 6000 AUV overcame these gaps and became an operational tool for the U.S. Navy.
\end{abstract}

\section{INTRODUCTION}

NAVOCEANO acquired the first of three full ocean depth Remote Environmental Monitoring UnitS (REMUS) 6000 vehicles in 2003 (Fig. 1). The second vehicle was acquired in 2004, and the third in 2005. The REMUS 6000 vehicles are 13 feet long, 2 feet in diameter, and weigh nearly 1,800 pounds. They are free-swimming, programmable, and redirectable AUVs that are full ocean depth rated $(6000 \mathrm{~m})$, making them capable of surveying nearly 97 percent of global ocean areas. REMUS 6000 vehicles, with their modular design, support deep-water characterization surveys. Sensors include side scan sonars, a sub-bottom profiler, electronic still cameras, conductivity-temperature-depth sensors, single-beam depth sensors, and acoustic doppler current profilers (ADCP). These sensors support acoustic and oceanographic characterization of areas of interest.

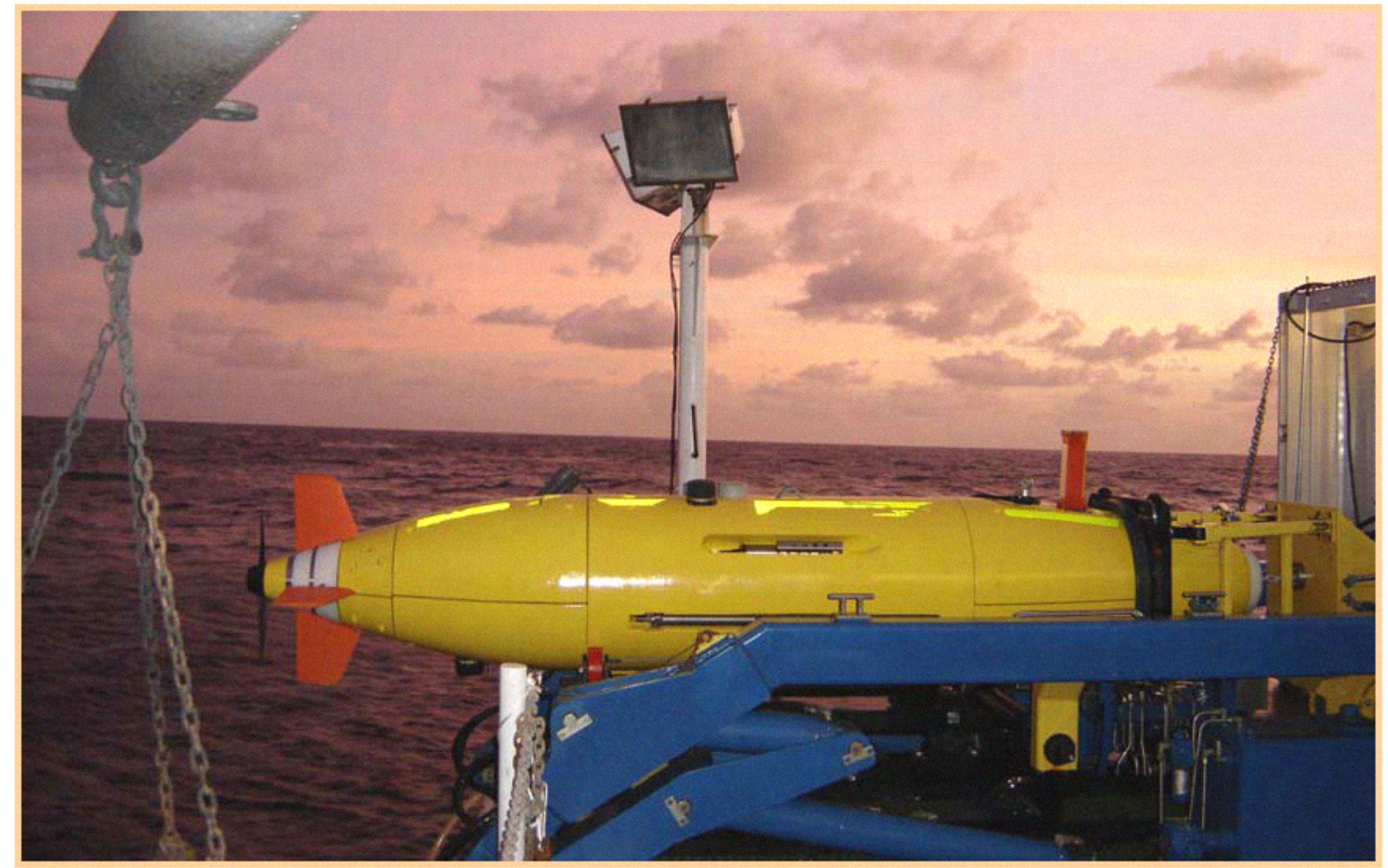

Fig. 1 REMUS 6000 vehicle in Launch and Recovery System, awaiting deployment. 
The REMUS vehicles are capable of operating in a greater range of sea-state conditions than the T-AGS 60 class survey ships, thereby improving NAVOCEANO's survey efficiency. In addition, the autonomy level of the REMUS vehicles allows these survey ships to work away from the vehicles (force multiplier), which serves to protect our personnel at sea by keeping manned systems out of harm's way. In addition, the vehicles are roll on/roll off systems that can be easily shipped worldwide. NAVOCEANO can deploy these systems to any of the six T-AGS 60 class ships (or ships of opportunity) to meet immediate Navy needs or emergent requirements.

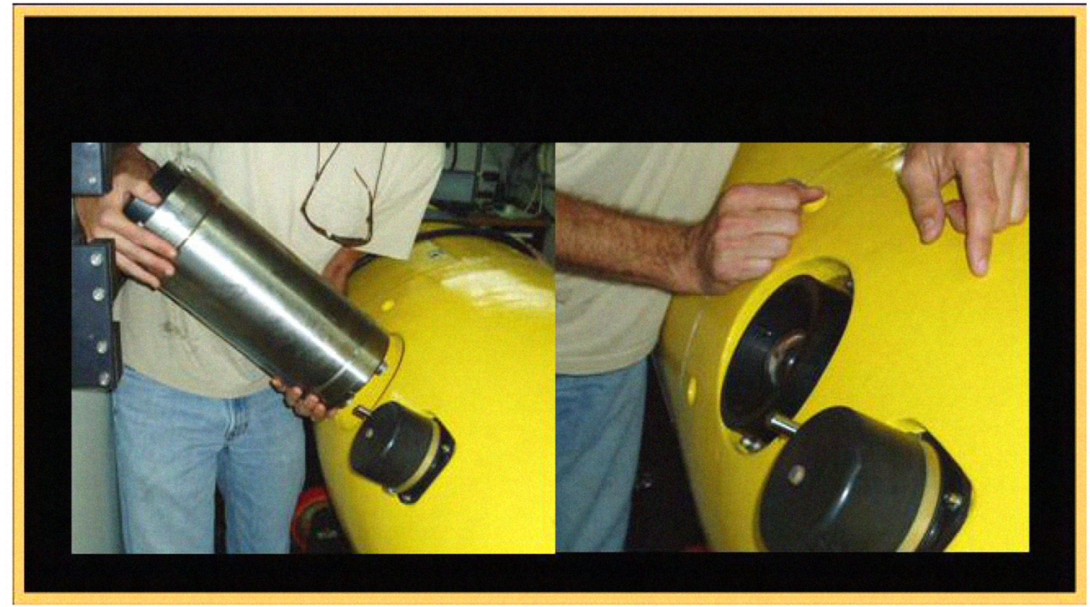

Fig. 2 The interchangeability of the electronic still camera and the sub-bottom profiler.

\section{TECHNOLOGY AdVANCES}

Advances in Lithium-Ion battery technology, sensor improvements, and inertial navigation have moved these systems to the forefront of NAVOCEANO surveying capability. The improvements in the lithium battery technology have expanded from the laptop world as more and more equipment takes advantage of this technology. This power source has allowed NAVOCEANO's REMUS 6000 systems to employ battery packs that now generate $11.2 \mathrm{~kW}$-hr of energy on a full charge. These batteries allow the REMUS 6000 vehicle to operate up to 22 hours with all systems collecting data.

One of the newer sensors onboard the REMUS 6000 vehicle is the sub-bottom profiler, developed by Omni Technologies Inc. This package is designed to exploit the 6000 's modular design, where sensors are interchangeable. The sub-bottom profiler module is easily swapped out with the Electronic Still Camera module on NAVOCEANO's vehicles, as shown in Fig. 2. This system was designed as an integrated package for the AUV. The sub-bottom profiler and camera system are similar in size and weight, eliminating complicated ballasting requirements. Both units also share a common platform electrical interface, alleviating cabling issues. The camera and the sub-bottom profiler can be interchanged on REMUS in less than 30 minutes. Both systems are 17 inches long and 7 inches in diameter.

The sub-bottom profiler is a high resolution and accurate sonar sensor that is based on a parametric design. Its operation allows for penetration of the sediment and the measuring of its properties. The sub-bottom profiler's parametric mode is the normal operating mode, providing bandwidth from $10 \mathrm{kHz}$ to $60 \mathrm{kHz}$ with a constant beam width of approximately $3^{\circ}$. As shown in Fig. 3 , sub-bottom data were collected in an area where a 55-gallon barrel was deployed. With the side scan data, the system demonstrates the ability of the vehicle to maneuver over the target and image the barrel. The subbottom profiler was operating at a 10-meter height and obtained bottom penetrations between 5 and 10 meters in sand sediments. Acoustic interference from the vehicle's acoustic modem is observed in the imagery. The frequency of the modem packets is defined by the operator and can be completely removed from the record.

The other interchangeable system on the REMUS 6000 is the Electronic Still Camera (ESC). The REMUS 6000 includes guest port switching on most externally connected systems. This feature allows the vehicle to recognize the system that is plugged into the port and

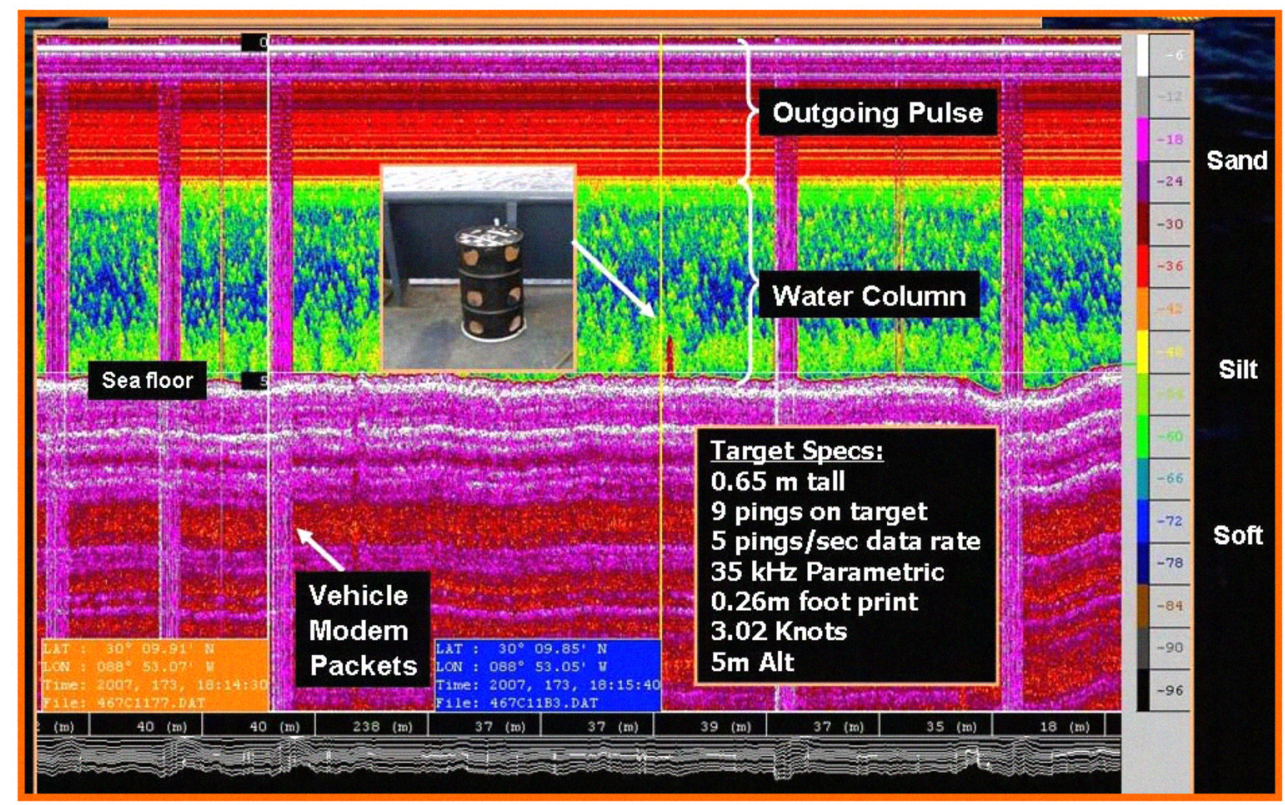

Fig. 3 Sub-bottom profile data snippet. 
configures it properly. New improvements have been made in the camera system for the REMUS 6000 system. These improvements begin with the camera lens. The lens is a Nikkor $15 \mathrm{~mm}$ lens that was designed for Nikon to be used by the recreational/professional underwater film photographic market. This wide-angle lens was designed exclusively for underwater use and is known for its ability to produce exceedingly sharp images. The camera is based on a $2 \mathrm{~K}$ x $2 \mathrm{~K}$ pixel (4 mega-pixel image size), Black/White Kodak KAI4021 CCD sensor. A 120 gigabyte hard disk provides storage. An ESC image (Fig. 4) was taken 6-8 meters above the bottom.

The REMUS 6000 is currently equipped with two side-look sonars. One of the REMUS 6000 vehicles (6000B) utilizes the EDGETECH 4200 FS side scan sonar system and is quickly becoming the new workhorse for NAVOCEANO in the collection of deep-ocean side scan sonar imagery. The REMUS $6000 \mathrm{C}$ vehicle

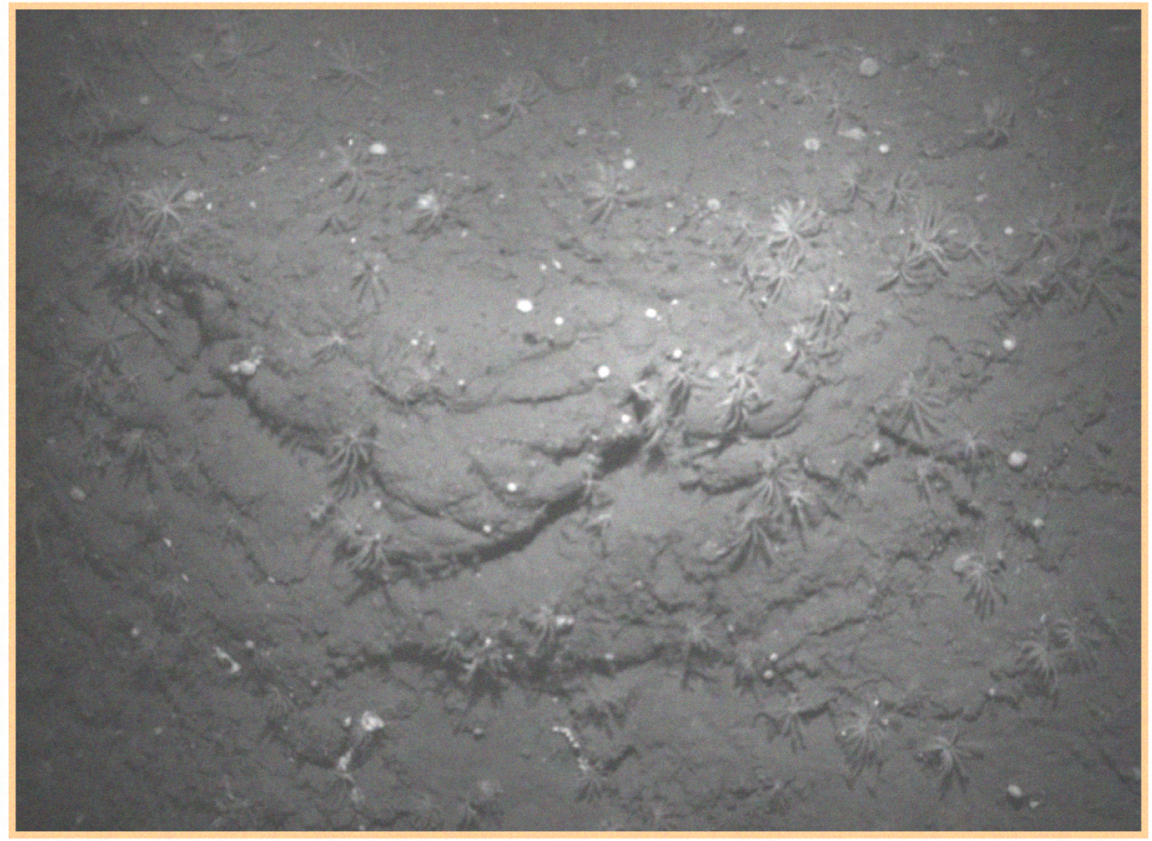

Fig. 4 Electronic still image of the bottom (altitude 3 - 4 meters). uses a Marine Sonics 300/900 kHz side-look sonar. The $4200 \mathrm{FS}$ is used primarily for large area surveys and records both the 120 $\mathrm{kHz}$ and $410 \mathrm{kHz}$ frequencies simultaneously. The Marine Sonics' frequency is user-selectable, recording at the $300 \mathrm{or} 900 \mathrm{kHz}$ frequency. Fig. 5 illustrates the EDGETECH side scan sonar imagery of Liberty ships in the Gulf of Mexico. Side scan sonar range was 25 meters, the operating altitude was 4 meters, and water depth was 20-25 meters.

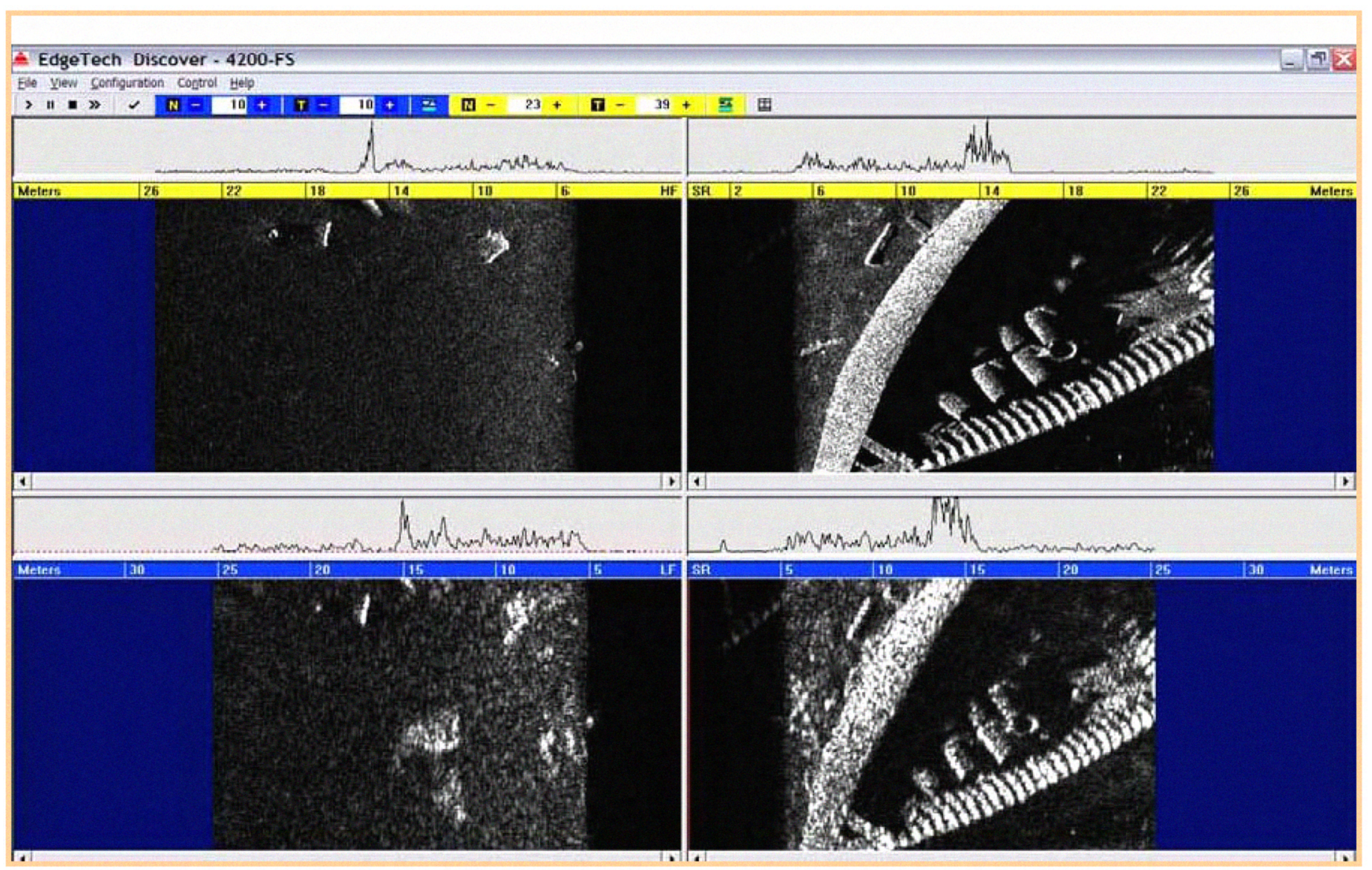

Fig. 5 EDGETECH sonar imagery of Liberty ships in the Gulf of Mexico. 
The REMUS 6000 vehicles are also equipped with an Inertial Navigation System (INS). This unit provides reliable heading, altitude, navigation, and vehicle dynamic data to the operator. The major component of the INS is a Kearfott T-16 inertial navigator. The INS utilizes inputs from its ring-laser-gyro, accelerometers, ADCP-Doppler velocity log, GPS, and acoustic Long Baseline (LBL) transponder systems. The INS navigator continuously utilizes all available data to determine the most likely instantaneous position at all times. When no other absolute navigation sources are present, the navigator continues to estimate position based on integrated velocities and accelerations. Typical error accumulations of less than 10 meters per hour have been seen in REMUS 6000 deployments. This technology allows the vehicle to operate in deep water for long periods without an LBL acoustic fix and greatly increases the effective area of transponder coverage. In many cases, the vehicle can leave the transponder net for extended periods, returning only occasionally to reset the navigation error.

Communications with AUVs, once the mission is complete and the vehicle has returned to the surface, has been a concern to the operators. A problem occurred when the ship was not in eyesight of the vehicle or in Wi-Fi range, and this problem needed a solution. With Iridium satellite communications (satellite network that covers the whole earth, including poles, oceans, and airways), the problem is solved. REMUS 6000 vehicles are equipped with Iridium chips, which enable it to call the ship and relay its position and vehicle status to the operators. Possible future improvements will include vehicle programming and redirections. The improvement in communications increases the comfort level of AUV operations and propels the REMUS vehicle into the mainstream of the Navy's surveying efforts.

\section{SUMMARY}

The REMUS 6000 systems are at the forefront of NAVOCEANO's deep ocean surveying effort. With the advances in sensors and navigation, higher quality data are collected, which translates into a better product for the Navy. Endurance and navigation accuracy improvements have enabled standoff operations, which enhances the ship safety and operational security. These vehicles augment the capabilities of NAVOCEANO's ships while providing an efficient means of collecting relevant data. With the advances in sensor development and navigation, AUVs are fast becoming the standard-bearer of the data collection effort for the Naval Oceanographic Office and the Navy.

Note: The inclusion of names of any specific commercial or academic product, commodity, or service in this paper is for informational purposes only and does not imply endorsement by the Navy or by NAVOCEANO. 